\title{
Neurodevelopmental outcomes in children with cerebellar malformations: a systematic review
}

\author{
MARIE-EVE BOLDUC BSC OT ${ }^{1}$ | CATHERINE LIMPEROPOULOS PHD OT(C) ${ }^{2}$ \\ 1 School of Physical and Occupational Therapy, McGill University, Montreal, Canada. 2 School of Physical and Occupational Therapy, Department of Neurology and \\ Neurosurgery, McGill University, Montreal, Canada. \\ Correspondence to Dr Catherine Limperopoulos at Montreal Children's Hospital, 2300 Tupper Street A-334, Montreal, Quebec, H3H 1P3, Canada. E-mail: \\ catherine.limperopoulos@mcgill.ca
}

\section{PUBLICATION DATA}

Accepted for publication 12th October 2008.

Published online 3rd February 2009.

LIST OF ABBREVIATIONS

DWM Dandy-Walker malformation

DWV Dandy-Walker variant

IVH Inferior vermis hypoplasia

MCM Mega cisterna magna

PCH Pontocerebellar hypoplasia

\section{ACKNOWLEDGEMENTS}

Marie-Eve Bolduc is supported by a McGill University Health Center Studentship award for her graduate studies. Dr Limperopoulos is supported by the Canadian Research Chairs Program.
Cerebellar malformations are increasingly diagnosed in the fetal period. Consequently, their consideration requires stressful and often critical decisions from both clinicians and families. This has resulted in an emergent need to understand better the impact of these early life lesions on child development. We performed a comprehensive literature search of studies describing neurodevelopmental outcomes of cerebellar malformations between January 1997 and December 2007. Overall, the data suggested that children with isolated inferior vermis hypoplasia (IVH) and mega cisterna magna (MCM) have a good developmental outcome, whereas children with molar tooth sign/Joubert syndrome, vermis hypoplasia, pontocerebellar hypoplasia (PCH) type II, and cerebellar agenesis experience moderate to severe global developmental delays. Reports for Dandy-Walker malformation (DWM) were conflicting; however, the presence of a normally lobulated vermis and the absence of associated brain anomalies were associated with a more favourable outcome. Finally, children with isolated cerebellar hypoplasia experienced fewer impairments. Important methodological limitations highlighted include a lack of standardized outcome measure use in $79 \%$ of studies and the predominant use of retrospective study designs $(85 \%)$, with $40 \%$ limited to case reports or case-series. In summary, rigorous outcome studies describing the spectrum of disabilities in survivors are urgently needed to accurately delineate the long-term neurodevelopmental consequences of cerebellar malformations.
Recent advances in neonatal intensive care and neuroimaging techniques, in particular magnetic resonance imaging, have greatly enhanced our ability to detect structural anomalies of the brain. This is particularly true for lesions in the posterior fossa. ${ }^{1,2}$ The incidence of posterior fossa malformations diagnosed in the newborn period is estimated to be 1 out of every 5000 live births. ${ }^{3}$ Fetal posterior fossa malformations on imaging are now among the most commonly diagnosed brain malformations in utero, though the actual prevalence is unknown. Advances in our ability to diagnose accurately cerebellar malformations have increased the need for a greater understanding of the impact of these early-life lesions on child function. Despite these advances, the long-term neurodevelopmental consequences of cerebellar malformations in children remain poorly defined. However, their consideration requires stressful and often critical decisions from both clinicians and families. This is particularly important in view of the fact that studies are now showing that up to $80 \%$ of parents choose to terminate their pregnancy after a prenatal diagnosis of a cerebellar malformation, even in the absence of rigorous outcomes data. ${ }^{4,5}$

In recent years, the traditional role of the cerebellum has been repeatedly challenged. The cerebellum, once underestimated as a simple centre for motor coordination and execution, is now increasingly recognized as a centre for higher cognitive functions as well. There is growing evidence in primate and adult literature to support an important role for the cerebellum in perceptual, linguistic, cognitive, and affective functions. ${ }^{1,6-9}$ In fact, Schmahmann and Sherman ${ }^{10}$ and Schmahmann ${ }^{11}$ have described the cerebellar cognitive affective syndrome in adults with 
lesions or malformations confined to the cerebellum. These patients were found to have a constellation of symptoms including cognitive, affective, and behavioural deficits. ${ }^{12}$ It has been hypothesized that the cerebellum acts as a modulator for all the cerebrocerebellar subsystems that control motor, sensory, cognitive, affective, and autonomic domains. ${ }^{10}$ Moreover, the cerebellum has been associated with deficits in spatial navigation, autism, and mutism, and with impaired ability to learn music. ${ }^{13}$

Despite this accumulating evidence, it is unclear whether these higher-order cognitive functions have been systematically evaluated in children with cerebellar malformations. Therefore, the objective of this paper was to summarize our current knowledge of the neurodevelopmental outcomes in children with cerebellar malformations. Studies describing cognitive, language, socialization, behavioural, or neuromotor outcomes published over the past decade were systematically reviewed.

\section{METHOD}

To delineate better the current impact of cerebellar malformations on child development, we performed a systematic review of the literature on neurodevelopmental outcomes in children with cerebellar malformations limited to studies published in the past 10 years (January 1997 to December 2007). Our systematic search was performed using PubMed, Medline, and CINAHL using the following keywords: cerebellar malformation; cerebellar dysgenesis; posterior fossa malformation; posterior fossa dysgenesis; cerebellar hypoplasia, cerebellar dysplasia, cerebellar agenesis; DWM; Dandy-Walker variant (DWV); Dandy-Walker complex; Dandy-Walker syndrome; vermis hypoplasia; rhombencephalosynapsis; pontocerebellar atrophy; pontocerebellar hypoplasia, (PCH); Joubert syndrome, molar tooth sign, development, and outcome. English-language studies describing neurodevelopmental outcomes in children $(0-18 y)$ were retained for this review. The reference list of selected articles was also searched.

\section{RESULTS}

The spectrum of dysgenetic abnormalities of the cerebellum is broad, ranging from subtle to very significant malformations. The most commonly described entity of cerebellar malformations is often referred to as the Dandy-Walker complex or continuum, a term used to characterize the different degrees of malformations of the cerebellar vermis and which includes DWM, DWV, or inferior vermis hypoplasia, (IVH), and mega-cisterna magna (described below). ${ }^{14,15}$ Other malformations that are summarized in the current review include the molar tooth sign, rhombencephalosynapsis, cerebellar hypoplasia/dysplasia, vermis hypoplasia, PCH, and vermis hypo- plasia. A total of 46 studies were reviewed based on our search strategy that specifically described neurodevelopmental outcomes in children with cerebellar malformations, and are summarized in Table I. An overall summary of the prevalence of developmental, cognitive, language, behavioural, and motor disabilities, as well as neurological abnormalities over our 10-year review period are provided in Table II. The frequency of occurrence of central nervous system (CNS) and extra-CNS findings are presented in Table III.

\section{Dandy-Walker malformation}

The most common and striking of these cerebellar malformations is known as the Dandy-Walker malformation (DWM). ${ }^{16} \mathrm{DWM}$ is characterized by partial or complete agenesis of the cerebellar vermis, cystic dilation of the fourth ventricle, and an enlarged posterior fossa combined with a superior displacement of the cerebellar hemisphere ${ }^{17}$ (Fig. 1). However, variations in the definition of DWM were evident over our 10-year review period. For instance, some authors included features such as the presence of hydrocephalus ${ }^{18,19}$ or the presence of communication between the posterior fossa cyst and the fourth ventricle as a fundamental criterion for the diagnosis of DWM. ${ }^{20}$ Conversely, some studies used the term Dandy-Walker complex to describe what most define as DWM. As a result, the generalizability of the data is limited and the importance of a universally accepted classification scheme for cerebellar malformation is a priority.

Overall, reports on the outcome of DWM were conflicting. Although one study reported that all children with DWM experienced some degree of cognitive impairment, ${ }^{19}$ other studies have reported a more favourable outcome. $^{17,20}$ Overall, up to one-third of survivors were reported to be developing normally. ${ }^{4,21,22}$ Specifically, Boddaert et al. ${ }^{17}$ compared the IQ of 21 children with DWM with and without normal vermis lobulation and showed that $82 \%$ of children in the former group had a normal IQ as opposed to none in the latter. Furthermore, among the subgroup with normal vermis lobulation and abnormal IQ, all children had associated CNS and extraCNS abnormalities. Similarly, Klein et al. ${ }^{20}$ divided 26 children into two groups, one with partial agenesis of the vermis with normal lobulation, and a second with severe vermis malformations. In the former group the majority (90\%) had a normal IQ and developmental quotients as opposed to none in the latter group. However, it is important to note that one of the two children with partial agenesis of the vermis who scored in the impaired range had fragile $\mathrm{X}$, and the other, severe periventricular leukomalacia resulting from being born preterm. It is also 
Table I: Summary of articles describing neurodevelopmental outcomes of children with cerebellar malformations over the 10-year review period

\begin{tabular}{|c|c|c|c|c|c|c|}
\hline Author & Year & Diagnosis & Study design & $n$ & Age range (mean/median) & $\begin{array}{l}\text { Standardized } \\
\text { outcome measures }\end{array}$ \\
\hline \multicolumn{7}{|c|}{ Dandy-Walker complex (DWC) } \\
\hline Forzano et al. ${ }^{5}$ & 2007 & DWC, MCM & Chart review & 56 & $2 \mathrm{~d}-5 \mathrm{mo}(1 \mathrm{mo})$ & No \\
\hline Poot et al..$^{23}$ & 2007 & DWM & Case report & 1 & $10 y(n / a)$ & No \\
\hline Limperopoulos et al. ${ }^{26}$ & 2006 & IVH & Cross-sectional & 19 & Range not specified (19.2mo) & Yes \\
\hline Abdel-Salam et al. ${ }^{24}$ & 2006 & DWM & Case report & 2 & $6-8 y(7 y)$ & No \\
\hline Long et al. ${ }^{25}$ & 2006 & DWM, DWV, MCM & Chart review & 86 & Range not specified $(96 \mathrm{mo})$ & No \\
\hline Has et al. ${ }^{22}$ & 2004 & DWM, DWV & Chart review & 78 & $3 \mathrm{mo}-5 \mathrm{y} 6 \mathrm{mo}(\mathrm{n} / \mathrm{a})$ & No \\
\hline Boddaert et al. ${ }^{17}$ & 2003 & DWM & Chart review & 21 & $9 m o-34 y$ & $Y_{e s}^{a}$ \\
\hline Klein et al. ${ }^{20}$ & 2003 & DWM & Chart review & 26 & Not specified (10y 6mo) & $Y_{e s}^{a}$ \\
\hline Kumar et al. ${ }^{18}$ & 2001 & DWS & Chart review & 42 & $9 \mathrm{mo}-12 \mathrm{y}(3 \mathrm{y} 10 \mathrm{mo})$ & No \\
\hline Ecker et al. ${ }^{4}$ & 2000 & DWC, DWV & Chart review & 99 & $6 w k(n / a)$ & No \\
\hline Kölble et al. ${ }^{21}$ & 2000 & DWM & Chart review & 10 & $4 w k-21 \mathrm{mo}(\mathrm{n} / \mathrm{a})$ & No \\
\hline Aletebi et al. ${ }^{19}$ & 1999 & DWM, MCM & Chart review & 15 & $23-50 \mathrm{mo}(\mathrm{n} / \mathrm{a})$ & $\mathrm{Yes}^{\mathrm{a}}$ \\
\hline Haimovici et al. ${ }^{27}$ & 1997 & $\mathrm{MCM}$ & Chart review & 15 & $0 d-9 m o(n / a)$ & No \\
\hline \multicolumn{7}{|c|}{ Molar-tooth sign/Joubert syndrome } \\
\hline Kumar et al. ${ }^{36}$ & 2007 & Joubert & Case report & 1 & $1 y$ & No \\
\hline Ray et al. ${ }^{39}$ & 2007 & Joubert & Case report & 1 & $7 \mathrm{mo}$ & No \\
\hline Braddock et al. ${ }^{41}$ & 2006 & Joubert & Cross-sectional & 21 & $32 \mathrm{mo}-19 \mathrm{y}(10 \mathrm{y} 5 \mathrm{mo})$ & Yes \\
\hline Romano et al. ${ }^{34}$ & 2006 & Molar tooth & Chart review & 13 & $2-16 y(n / a)$ & No \\
\hline Hodgkins et al. ${ }^{37}$ & 2004 & Joubert & Chart review & 18 & $3 \mathrm{mo}-21 \mathrm{y}(10 \mathrm{y} 11 \mathrm{mo})$ & $Y_{e s}{ }^{a}$ \\
\hline Kumandas et al. ${ }^{30}$ & 2004 & Joubert & Cross-sectional & 7 & $4 d-8 y(n / a)$ & No \\
\hline Torres et al. ${ }^{38}$ & 2001 & Joubert & Case report & 1 & $40 \mathrm{mo}$ & $\mathrm{Yes}^{\mathrm{a}}$ \\
\hline Fennell et al. ${ }^{32}$ & 1999 & Joubert & Cross-sectional & 51 & $11 \mathrm{mo}-17 \mathrm{y}(\mathrm{n} / \mathrm{a})$ & Yes \\
\hline Maria et al..$^{33}$ & 1999 & Joubert & Cross-sectional & 61 & 1y $4 \mathrm{mo}-17 \mathrm{y}(7 \mathrm{y} 6 \mathrm{mo})$ & No \\
\hline Gitten et al. ${ }^{35}$ & 1998 & Joubert & Cross-sectional & 32 & $14-204 \mathrm{mo}(68.7 \mathrm{mo})$ & Yes \\
\hline Steinlin et al. ${ }^{40}$ & 1997 & Joubert & Chart review & 19 & $1 y 6 m o-37 y(n / a)$ & No \\
\hline \multicolumn{7}{|c|}{ Rhombencephalosynapsis (RCS) } \\
\hline Chemli et al. ${ }^{42}$ & 2007 & RCS & Case report & 1 & $3 y 6 \mathrm{mo}$ & No \\
\hline Odemis et al. ${ }^{48}$ & 2003 & RCS & Case report & 1 & $8 \mathrm{mo}$ & No \\
\hline Jellinger $^{47}$ & 2002 & RCS & Case report & 1 & $7 y$ & No \\
\hline Toelle et al. ${ }^{43}$ & 2002 & RCS & Cross-sectional & 9 & 1y 6mo-6y (n/a) & No \\
\hline Danon et al. ${ }^{46}$ & 2000 & RCS & Case report & 1 & $5 y$ & No \\
\hline Utsunomiya et al. ${ }^{44}$ & 1998 & RCS & Case report & 2 & Infancy $-4 y$ & No \\
\hline Aydingoz et al. ${ }^{45}$ & 1997 & RCS & Case report & 1 & $17 \mathrm{mo}$ & No \\
\hline \multicolumn{7}{|c|}{ Cerebellar hypoplasia/dysplasia } \\
\hline Tavano et al. ${ }^{54}$ & 2007 & Hypoplasia, dysplasia & Cross-sectional & 27 & $3-34 y(11 y$ 1mo) & Yes \\
\hline Tavano et al. ${ }^{57}$ & 2007 & Dysgenesis & Chart review & 5 & $2-11 y(n / a)$ & $Y_{e s}{ }^{a}$ \\
\hline Ventura et al. ${ }^{56}$ & 2006 & Hypoplasia & Chart review & 14 & $4-20 y(n / a)$ & $Y_{e s}{ }^{a}$ \\
\hline Yapici et al. ${ }^{53}$ & 2005 & Hypoplasia & Chart review & 2 & $5-12 y$ & No \\
\hline McCollom et al. ${ }^{55}$ & 2003 & Hypoplasia & Case report & 1 & $6 \mathrm{mo}$ & No \\
\hline Wassmer et al. ${ }^{52}$ & 2003 & Hypoplasia & Chart review & 45 & Not specified (children) & No \\
\hline Soto-Ares et al. ${ }^{51}$ & 2000 & Dysplasia & Chart review & 46 & $10 d-14 y(n / a)$ & No \\
\hline \multicolumn{7}{|l|}{ Vermis hypoplasia } \\
\hline Bruck et al. ${ }^{58}$ & 2000 & Hypoplasia & Case report & 2 & $2 y$ and $9 y$ & No \\
\hline Koutsouraki et al. ${ }^{59}$ & 2007 & Hypoplasia & Case report & 1 & $15 y$ & No \\
\hline \multicolumn{7}{|c|}{ Pontocerebellar hypoplasia type II (PCH) } \\
\hline Steinlin et al. ${ }^{60}$ & 2007 & $\mathrm{PCH}$ & Chart review & 21 & $4 \mathrm{mo}-11 \mathrm{y} 2 \mathrm{mo}(49 \mathrm{mo})$ & No \\
\hline Dilber et al. ${ }^{63}$ & 2002 & $\mathrm{PCH}$ & Case report & 2 & $30 \mathrm{mo}$ and $17 y$ & No \\
\hline Sans-Fito et al. ${ }^{65}$ & 2002 & $\mathrm{PCH}$ & Case report & 1 & $3 y$ & No \\
\hline Coppola et al. ${ }^{64}$ & 2000 & $\mathrm{PCH}$ & Case report & 2 & $18 \mathrm{mo}$ and $5 y$ & No \\
\hline
\end{tabular}


Table I: Continued

\begin{tabular}{lllllll}
\hline Author & Year & Diagnosis & Study design & $n$ & Age range (mean/median) & $\begin{array}{l}\text { Standardized } \\
\text { outcome measures }\end{array}$ \\
\hline $\begin{array}{l}\text { Cerebellar agenesis } \\
\text { Titomanlio et al. }{ }^{67}\end{array}$ & 2005 & Agenesis & Case report & 1 & $17 y$ & No \\
Gardner et al. ${ }^{68}$ & 2001 & Agenesis & Chart review & 5 & $2-17 y(\mathrm{n} / \mathrm{a})$ & No \\
\hline
\end{tabular}

aStandardized outcome measures extracted from chart review. DWC, Dandy-Walker complex; DWM, Dandy-Walker malformation; DWS, Dandy-Walker syndrome; DWV, Dandy-Walker variant; IVH, inferior vermis hypoplasia; MCM, mega cisterna magna; $\mathrm{PCH}$, pontocerebellar hypoplasia; RCS, rhombencephalosynapsis; $\mathrm{n} / \mathrm{a}$, not available.

Table II: Summary of developmental/cognitive delays, language, behavioural deficits, and neurological abnormalities in children with cerebellar malformations

\begin{tabular}{lllll}
\hline Diagnostic group & $\begin{array}{l}\text { Developmental/ } \\
\text { cognitive delay (\%) }\end{array}$ & $\begin{array}{l}\text { Language } \\
\text { deficits (\%) }\end{array}$ & $\begin{array}{l}\text { Social/behavioural } \\
\text { deficits (\%) }\end{array}$ & $\begin{array}{l}\text { Neurological } \\
\text { abnormalities (\%) }\end{array}$ \\
\hline Molar tooth sign/Joubert syndrome & 100 & 100 & 100 & 100 \\
Vermis hypoplasia & 100 & 100 & 100 & $20-100$ \\
Pontocerebellar hypoplasia type II & 100 & $\mathrm{n} / \mathrm{a}$ & $\mathrm{n} / \mathrm{a}$ & 100 \\
Cerebellar agenesis & 100 & 100 & $\mathrm{n} / \mathrm{a}$ & 80 \\
Dandy-Walker malformation & $67-100$ & 50 & $\mathrm{n} / \mathrm{a}-71$ & $50-100$ \\
Cerebellar hypoplasia/dysplasia & $53-87$ & $6-100$ & 61 & $67-100$ \\
Rhombencephalosynapsis & 56 & 25 & $\mathrm{n} / \mathrm{a}$ & 94 \\
Dandy-Walker variant & 46 & $\mathrm{n} / \mathrm{a}$ & 23 & 100 \\
Isolated inferior vermis hypoplasia & 23 & 23 & $\mathrm{n} / \mathrm{a}$ & 23 \\
Mega cisterna magna & $0-8$ & $\mathrm{n} / \mathrm{a}$ & 0 \\
\hline
\end{tabular}

$\mathrm{n} / \mathrm{a}$, not available.

Table III: Summary of central nervous system (CNS) and non-CNS anomalies in children with cerebellar malformations

\begin{tabular}{lll}
\hline & $\begin{array}{l}\text { Associated } \\
\text { CNS } \\
\text { anomalies (\%) }\end{array}$ & $\begin{array}{l}\text { Associated } \\
\text { non-CNS } \\
\text { anomalies (\%) }\end{array}$ \\
Diagnostic group & $20-88$ & $29-47$ \\
\hline $\begin{array}{l}\text { Cerebellar hypoplasia/dysplasia } \\
\text { Rhombencephalosynapsis }\end{array}$ & 56 & 25 \\
$\begin{array}{l}\text { Dandy-Walker malformation } \\
\text { Dandy-Walker variant }\end{array}$ & $43-67$ & $9-75$ \\
$\begin{array}{l}\text { Pontocerebellar hypoplasia } \\
\text { type II }\end{array}$ & $35-71$ & $64-65$ \\
$\begin{array}{l}\text { Mega cisterna magna } \\
\text { Molar tooth sign/Joubert } \\
\text { syndrome }\end{array}$ & 55 & $\mathrm{n} / \mathrm{a}$ \\
$\begin{array}{l}\text { Cerebellar agenesis } \\
\text { Vermis hypoplasia }\end{array}$ & $0-38-66$ & $18-62$ \\
\hline
\end{tabular}

$\mathrm{n} / \mathrm{a}$, not available.

worthy of mention that all children with severe vermis malformations had associated cerebral anomalies, three of which had agenesis of the corpus callosum.

Language and communication abilities in survivors of DWM have not been well described. In fact, language defi- cits were only described in a single chart review and two case reports. ${ }^{19,23,24}$ Conversely, neurological abnormalities have been reported in up to $50 \%$ of survivors ${ }^{4,18,21,22}$ and included hypotonia (50\%), ${ }^{21,23,24}$ signs of cerebellar dysfunction (not further described; 42\%), ${ }^{18}$ and hemiparesis (5\%). ${ }^{18}$

Associated anomalies in both the CNS and other systems have been reported in up to $86 \%$ of children. ${ }^{4}$ Specifically, CNS abnormalities have been described in 13 to $67 \%$ of cases, ${ }^{17-22}$ with the most common anomaly being ventriculomegaly, observed in 36 to $67 \%$ of children. ${ }^{4,17,21,22}$ Other common CNS malformations included agenesis of corpus callosum, reported in 5 to $50 \%$ of children. ${ }^{5,18,21,23}$ Interestingly, agenesis of the corpus callosum was observed in 60 to100\% of the children with abnormal vermis lobulation. ${ }^{17,20}$ In studies where comparison of children with isolated DWM and those who had associated CNS anomalies was possible, we found that all children with DWM who were developing normally had no associated CNS malformations. ${ }^{17,20,21}$ Conversely, all but one with developmental delays had associated CNS anomalies or epilepsy. ${ }^{17,19-21}$ However, many studies clustered those with and without concurrent CNS findings, and therefore further analysis was not possible. Extra-CNS 


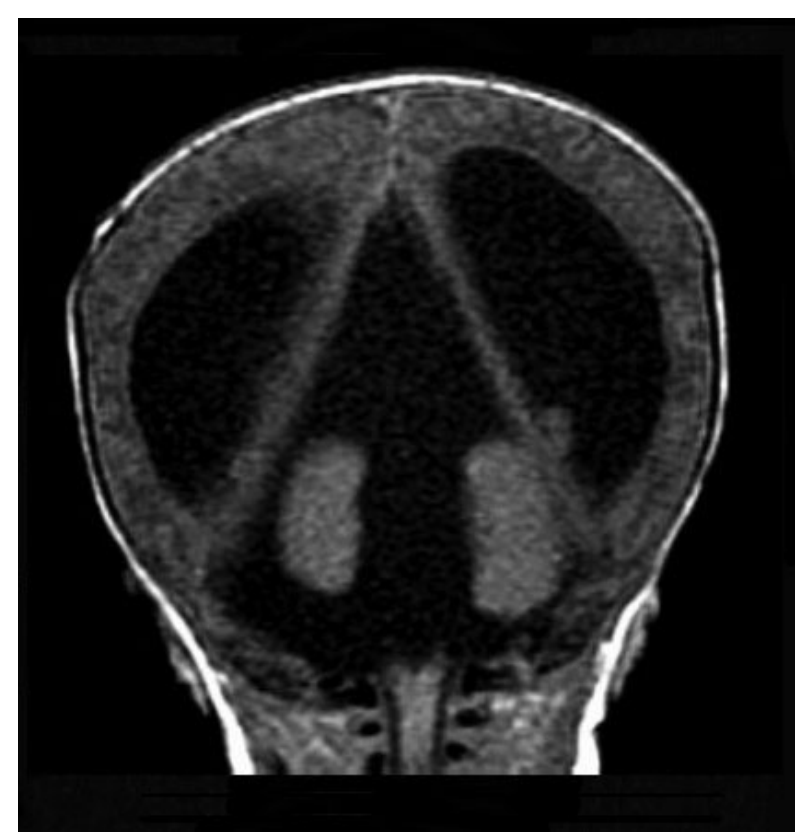

Figure 1: T1-weighted coronal magnetic resonance image of a term infant with Dandy-Walker malformation, characterized by hypoplasia of the cerebellar vermis, massive cystic dilation of the fourth ventricle, and elevated tentorium.

anomalies were less common, and were reported in 9 to $44 \%$ of children ${ }^{18,22}$ and included structural heart defects, renal, extremity and facial anomalies, and single umbilical artery. ${ }^{4,17,18,21-24}$

Two studies in the literature have collectively described the outcome of children with DWM and DWV without differentiating between the two diagnostic groups. Forzano et al. ${ }^{5}$ compared the outcome in 34 children with isolated and non-isolated DWM and DWV diagnosed prenatally. Interestingly, $68 \%$ of parents elected to terminate the pregnancy. Among survivors, 40\% were developing normally, of which half were diagnosed with associated syndromes ${ }^{5}$ including occipital encephalocoele. In a second study $^{25}$ all children with atypical development had associated anomalies, however, the presence of CNS anomalies did not predict a poorer outcome. Associated (CNS and non-CNS) anomalies were reported in $83 \%$ of cases. ${ }^{25}$

In summary, available evidence on the outcome of DWM suggests a more favourable neurodevelopmental outcome in children with no associated supratentorial findings and in those with a normally lobulated vermis.

\section{Dandy-Walker variant}

DWV has been used to describe a combination of cystic dilation of the fourth ventricle and hypoplastic cerebellar vermis in the absence of an enlargement of the posterior fossa. ${ }^{14}$ However, in recent years, it has been strongly advocated that the term DWV be abandoned altogether, given its multiple and variable definitions. ${ }^{14}$ To date, these inconsistencies have prevented the meaningful comparison of diagnosis and outcome among published series, thereby compromising accurate prognostication. As such, it is now strongly recommended that the term DWV be abandoned altogether. However, for the purposes of this review, we have summarized the literature that has described the outcome of DWV to date. Some reports indicate that more than half of children with DWV were developing normally. ${ }^{4}$ On the other hand, in a study by Has et al., ${ }^{22}$ all children with DWV experienced neurological sequelae, including microcephaly in 21\%. Associated anomalies (CNS and non-CNS) have been described in up to $71 \%$ of children with DWV, with the most common being ventriculomegaly $(27-71 \%)^{4,22}$ and agenesis of corpus callosum (14\%).,22 Extra-CNS anomalies have also been reported in up to $65 \%,{ }^{4,22}$ with cardiac, renal, extremity, and facial anomalies occurring most frequently., ${ }^{4,22}$

\section{Inferior vermis hypoplasia}

IVH is characterized by partial absence of the inferior portion of the cerebellar vermis with normal- or near normalshaped cerebellar hemispheres, a normal-sized posterior fossa without obvious cystic lesions, and normal supratentorial structures $^{26}$ (Fig. 2). IVH represents an arrested, incomplete downward growth of the vermis, leaving an enlarged midline cerebrospinal-fluid space, which may be mistaken for a cystic lesion. It is important to note that the diagnostic entity of $\mathrm{IVH}$ continues to be inconsistently used. For example, some investigators consider this lesion a normal variant, whereas others have used the term DWV interchangeably, even in the absence of a cystic fourth ventricle and with a normal-sized posterior fossa.

Normal development was reported in $77 \%$ of children with isolated $\mathrm{IVH}^{26}$ In the subgroup of children (23\%) with isolated IVH who had delayed development, gross and fine motor disabilities, as well as social and communication deficits, were reported. Furthermore, $15 \%$ of these children were found to have behavioural problems, particularly symptoms of disruptive behaviour. ${ }^{26}$ Moreover, $23 \%$ of the children with IVH were found to be hypotonic on neurological examination.

\section{Mega cisterna magna}

MCM is characterized by an enlarged cisterna magna with a normal fourth ventricle and cerebellar hemispheres and vermis $^{14}$ (Fig. 3). The developmental outcome of children with MCM was generally described as favourable, with the majority of children (92-100\%) with isolated MCM 


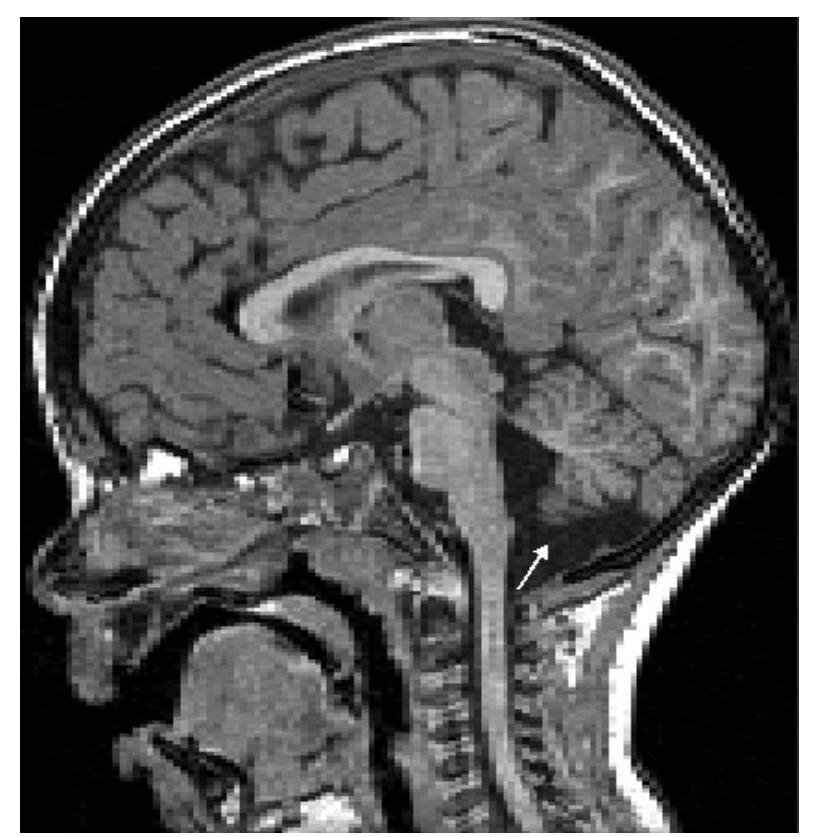

Figure 2: T1-weighted magnetic resonance image of the midline sagittal view illustrating incomplete downgrowth of the vermis (arrow) in a 18-month-old child with inferior vermian hypoplasia.

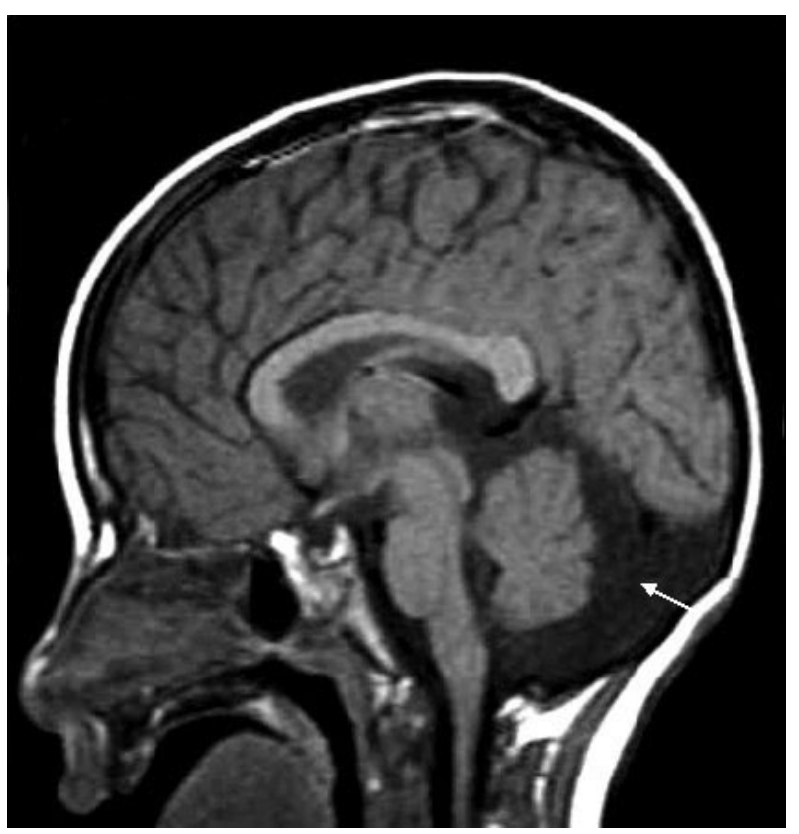

Figure 3: T1-weighted sagittal magnetic resonance image showing an enlarged cisterna magna (arrow) and normal fourth ventricle and cerebellar hemispheres and vermis in a 2-year-old child with mega cisterna magna. developing normally. ${ }^{5,25,27}$ In fact, on the basis of medical record reviews only one patient was presenting with delayed motor development. ${ }^{25}$ However, available data in adults with MCM suggest that higher cognitive functions and language abilities, such as verbal memory and fluency, executive functions and semantic fluency, may be impaired in this population. ${ }^{28}$ It is possible that more subtle deficits may be undiagnosed because of the lack of in-depth neuropsychological testing performed in the paediatric studies that were reviewed, or that some of these higher cognitive deficits present in later life.

More than two-thirds of children with MCM and associated CNS (e.g. ventriculomegaly) and non-CNS anomalies (e.g. orthopaedic malformations) ${ }^{5}$ were reported to be developing normally ${ }^{5,25}$ on the basis of medical chart reviews. In the remaining one-third, the spectrum of disability included cognitive and language delay as well as delayed motor development and neurological abnormalities (e.g. cerebellar ataxia). ${ }^{25}$ Only one patient had severe cognitive impairment; however, this patient was also diagnosed with cytomegalovirus infection and therefore deficits cannot be directly linked to the cerebellar malformation. Ventriculomegaly was the most common CNS finding associated with MCM, reported in 46 to $66 \%$ of children, ${ }^{5,25}$ whereas a renal defect was the most frequent nonCNS anomaly described in approximately one-third. ${ }^{5,25}$ Agenesis of the corpus callosum and cardiac and liver anomalies were also reported but these were less frequent $(4 \%)^{25}$

In summary, the presence of concomitant CNS anomalies in children with MCM was associated with a poorer prognosis albeit most children were developing normally and impairments were found to be mild in severity.

\section{Molar tooth sign/Joubert syndrome}

The molar tooth sign is characterized by an abnormally deep interpeduncular fossa, enlarged superior cerebellar peduncles that are more horizontally oriented, and a hypoplastic cerebellar vermis ${ }^{29}$ (Fig. 4). Joubert syndrome is the most known syndrome typified and is associated with developmental delays, hypotonia, breathing anomalies, abnormal eye movement, and facial dysmorphia. ${ }^{30}$ More than eight different types of Joubert syndrome-related disorders have been identified and were found to have various genotypes and phenotypes. ${ }^{31}$ Although the different types of Joubert syndrome-related disorders may have diverse outcomes, it was not possible in the context of the literature reviewed to identify the specific impact of each type of Joubert syndrome on neurodevelopmental outcome.

Nevertheless, available evidence suggests that impaired cognitive function or developmental delay was present in all children, with the majority experiencing severe 


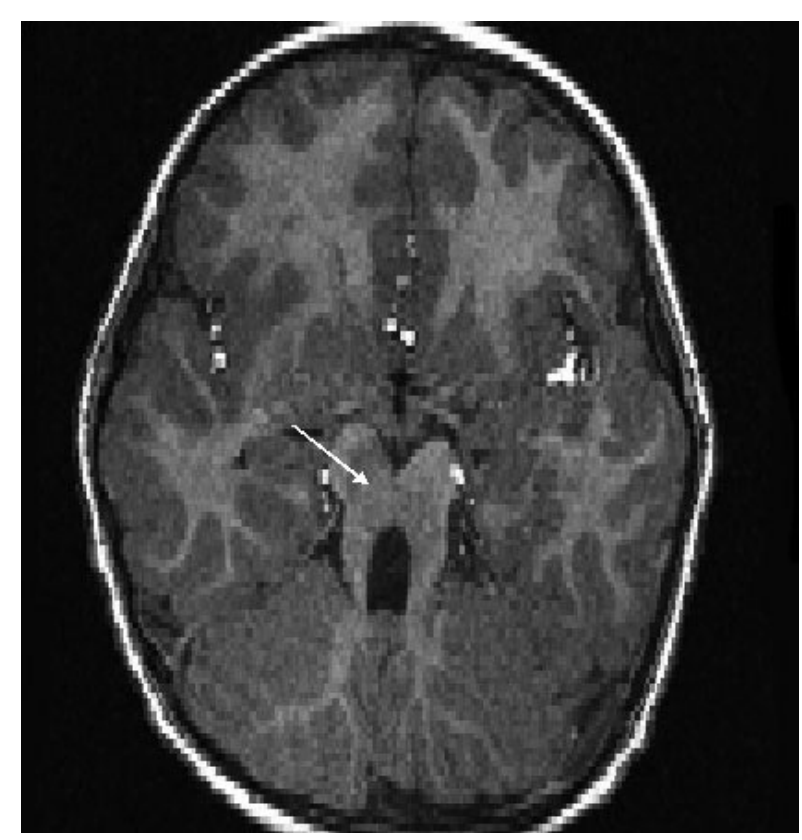

Figure 4: T1-weighted axial magnetic resonance image at the level of the brainstem showing deep interpeduncular cistern (as a result of reduced pyramidal decussation (arrow)), thick superior cerebellar peduncles, and enlarged fourth ventricle representing the molar tooth sign in a 3-year old with child Joubert syndrome.

disability. ${ }^{30,32-39}$ Moreover, in addition to delayed developmental milestones and impaired developmental quotients, immediate and delayed memory, conceptual development, perceptual discrimination, and daily living skills were

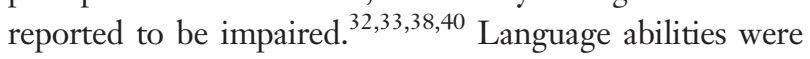
also affected in all children with molar tooth sign or Joubert syndrome. $9,32,34,37,38,40,41$ In particular, deficits in expressive language, ${ }^{38}$ verbal fluency, ${ }^{33}$ and vocabulary were noted. ${ }^{32,34}$ Furthermore, approximately half of children with Joubert syndrome were found to have impaired concept development, as evaluated by the Bracken Basic Concept Scale. ${ }^{32}$ Behavioural and social problems were also found to be prevalent in two studies. ${ }^{32,38}$ In fact, $100 \%$ of parents reported their children as being demanding or strong-willed the children were also described as hyperactive (50\%) or aggressive (25\%). Additionally, in a case report a child was reported to have significant social deficits, ${ }^{39}$ and two other children had behavioural difficulties (tendency to aggression). ${ }^{40}$ Moreover, significant motor delays were frequently reported. . $^{30,32,34,37,38,40,41}$

Neurological impairments were present in all cases, ${ }^{30,32,34,40}$ and were characterized by ataxia $(100 \%),{ }^{30,32}$ hypotonia (97-100\%), $30,33,34,36-40$ and oculomotor disturbances $(42-77 \%){ }^{30,33,34,36,38,40}$ In addition, visuomotor deficits were also found to be prevalent. ${ }^{32}$ Associated CNS anomalies were less prevalent, with the most common CNS

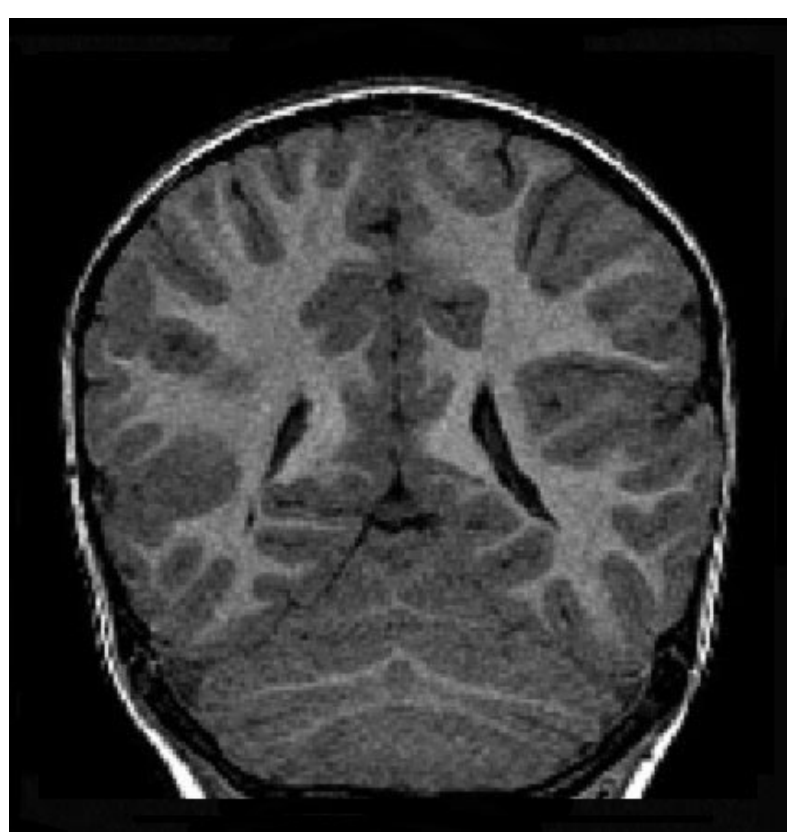

Figure 5: T1-weighted coronal magnetic resonance image of a 3-year-old child with rhombencephalosynapsis, demonstrating a complete absence of the vermis and fusion of the cerebellar hemispheres.

abnormality being dysgenesis of the corpus callosum in 5 to $29 \%$ of children. ${ }^{30,33,37}$ Atrophy of the cerebrum, ${ }^{33}$ anomalies of the mesencephalon ${ }^{30}$ and of the pontomesencephalic junction, ${ }^{33}$ brainstem hypoplasia, ${ }^{37}$ dilation of the ventricular system, ${ }^{37}$ as well as delayed myelination ${ }^{33}$ and whitematter lesions ${ }^{37}$ were also reported. The most common extra-CNS anomalies included breathing abnormalities $(38-86 \%),{ }^{30,33,34,37}$ facial dysmorphism $(71 \%),{ }^{30,38}$ and extremity malformations $(8-43 \%){ }^{30,33,34,37}$

\section{Rhombencephalosynapsis}

Rhombencephalosynapsis is considered a rare cerebellar malformation that includes agenesis of the cerebellar vermis and fusion of the cerebellar hemispheres ${ }^{14}$ (Fig. 5). Data describing cognitive development in this population have been conflicting: some have reported normal cognitive abilities ${ }^{42,43}$ whereas others have reported severe learning disability* and developmental delay. ${ }^{44-47}$ Neuromotor impairments were reported in all case reports of rhombencephalosynapsis, ${ }^{42-48}$ including delayed motor development ${ }^{44,48}$ hypotonia, ${ }^{44,48}$ cerebral palsy ${ }^{43}$ decreased balance, ${ }^{46}$ and oculomotor disturbances. ${ }^{44,46}$ Language deficits were reported in a single case report. ${ }^{44}$ Irritability was reported in one child. ${ }^{45}$

*North American usage: mental retardation. 
Importantly, CNS abnormalities have also been reported in the majority of cases with rhombencephalosynapsis, ${ }^{42-44,46,47}$ including agenesis or thinning of the corpus callosum, hydrocephalus, and ventriculomegaly. Non-CNS malformations included facial dysmorphism $^{42,45}$ as well as extremity ${ }^{45}$ anomalies. Interestingly, the presence of CNS or extra-CNS malformations was not found to be predictive of a poorer outcome in this population. However, it is important to note that the majority of studies did not use standardized outcome measures.

\section{Cerebellar hypoplasia and dysplasia}

Cerebellar hypoplasia is characterized by incomplete or underdevelopment of the cerebellum ${ }^{49}$ whereas cerebellar dysplasia is characterized by an abnormality in maturation of tissue cells. ${ }^{50}$ The studies presented herein described the outcome of children with focal cerebellar hemispheric hypoplasia and global cerebellar hypoplasia. Children with vermis hypoplasia were also included if the study design did not allow separate analysis. It is important to note that the clustering of children with various types of cerebellar hypoplasias is a recurrent limitation in the available literature. Consequently, this affects our ability to characterize reliably the outcome of the different types of hypoplasias or dysplasias. For example, unilateral hypoplasias are believed to be the result of a prenatal lesion rather than being true malformations. ${ }^{1}$ However, whenever possible, we present a summary of the outcome of children with unilateral and bilateral hypoplasia separately.

Available evidence showed that well over half of children $(53-87 \%)^{51-54}$ were found to have developmental delay or cognitive impairments, among whom over one-third (38\%) had severe deficits. ${ }^{54}$ Although one case report described a child with 'normal neural development' at 6-month followup, no information was provided. ${ }^{55}$ Overall, language deficits were found to be prevalent in the majority of studies but were reported as infrequent by others (6-95\%), ${ }^{51-54,56}$ and the levels of disability ranged from mild impairment (76\%) to severe impairment or total absence of language development (19\%). ${ }^{54}$ Furthermore, 5 to $20 \%$ were reported to present with autistic features, ${ }^{52,54} 81 \%$ were found to have an impaired affect, ${ }^{55}$ and $71 \%$ social or behavioural difficulties. Delayed motor development was variable, observed in 18 to $90 \%$ of children. ${ }^{51-54,56}$

Neurological abnormalities were described in up to $100 \% .^{53}$ They included increased tone $(58 \%),{ }^{52}$ ataxia and/or decreased coordination (12-49\%), ${ }^{51,52,54}$ hypotonia (49-93\%), ${ }^{51,52,54,56}$ oculomotor disturbances (35-57\%), ${ }^{51,52,56}$ and abnormal movements such as tremor, dysdiadochokinesis, or head titubation $(9-100 \%) .{ }^{52,54}$ It is noteworthy that all reported cases had associated cerebral anomalies in one of the larger studies: ${ }^{52} 22 \%$ suffered from cerebral atrophy or periventricular leukomalacia; $20 \%$ were microcephalic; $16 \%$ had neuronal migrational defects; and $11 \%$ had anomalies of corpus callosum. Moreover, 20\% of cases had associated syndromes or disorders. ${ }^{52}$ Associated non-CNS anomalies included facial dysmorphism ${ }^{51,56}$ and skeletal $^{52}$ and kidney malformations. ${ }^{51}$

In two chart reviews of children with isolated cerebellar dysgeneses, ${ }^{56,57} 40$ to $71 \%$ were described to have normal cognitive development, $40 \%$ showed mild impairment, and $20 \%$ moderate impairment. ${ }^{57}$ Moreover, language skills were affected in all children, with $80 \%$ being mildly impaired and 20\% moderately impaired. ${ }^{57}$ Affect was reported to be normal in $60 \%$, whereas $20 \%$ had a mild impairment and $20 \%$ a moderate impairment. ${ }^{57}$ In addition, 14 to $80 \%$ of the children were reported to have emotional, social, or behavioural difficulties. ${ }^{56,57}$ Finally, 71 to $100 \%$ had motor impairments as well. ${ }^{56,57}$

In the subset of articles in which we were able to distinguish between unilateral and bilateral hypoplasia/ dysplasia, bilateral cerebellar lesions were found to be associated with a poorer outcome. Specifically, children with bilateral cerebellar hypoplasia experienced a high prevalence of cognitive/developmental delay (60-100\%) compared with those children with unilateral cerebellar lesions (17-50\%). ${ }^{51,54,56,57}$ Similarly, language impairments were reported in 44 to $89 \%$ in the former group versus 17 to $100 \%$ in the latter group, ${ }^{51,54,56,57}$ Behavioural difficulties, neurological deficits, and associated CNS anomalies were also reported more frequently in children with bilateral cerebellar hypoplasia and dysplasia. ${ }^{51,54-57}$

In summary, outcome data on children with hypoplasia and dysplasia of the cerebellum are inconsistent. The presence of a large and variable spectrum of disability among survivors described in the present studies could be explained by the important differences in the topography and severity of the lesions. However, children with isolated hypoplasia of the cerebellar hemispheres appear to have a more favourable prognosis. ${ }^{57}$

\section{Vermis hypoplasia}

Vermis hypoplasia is characterized by incomplete development or underdevelopment of the cerebellar vermis. A subgroup of five children with partial or complete hypoplasia of the cerebellar vermis was reported by Tavano et al. ${ }^{54}$ All children presented with developmental delays: $80 \%$ with severe delay and 20\% with moderate deficits. Moreover, language skills were affected in all children, with $80 \%$ presenting with severe deficits and $20 \%$ with complete absence of language skills. In addition, all children showed impairment in behaviour modulation. Motor development was found to be delayed 
in all children but to a lesser degree, with $80 \%$ displaying a moderate deficit and $20 \%$ a severe deficit. Neurological abnormalities included hypotonia $(100 \%)$, ataxia $(80 \%)$, and intention tremor (20\%).

Additionally, Bruck et al. ${ }^{58}$ reported a case of two siblings with vermis hypoplasia, one of whom was described as having normal cognitive skills. However, language and motor skills were impaired in both children and both presented with hypotonia. Furthermore, severe cognitive and language impairments as well as motor disabilities were described in a case report of a 15 -year-old male with cerebellar vermis hypoplasia. ${ }^{59}$ Neurological findings included hypotonia, oculomotor dysfunction, and ataxia. The presence of CNS (megalocephaly) and extra-CNS (e.g. micrognathy and syndactyly) abnormalities were also reported.

Finally, three patients with vermis hypoplasia were described by Ventura et al., ${ }^{56}$ of which two were found to have impaired cognition and one was reported to have motor delay and anxiety.

In summary, available data suggest that the majority of children with partial or near-complete hypoplasia of the vermis present with global developmental delay, as well as language, motor, and neurological disabilities.

\section{Pontocerebellar hypoplasia}

$\mathrm{PCH}$ is a heterogeneous group of conditions characterized by hypoplasia of the cerebellum and the ventral pons. ${ }^{60} \mathrm{It}$ can be divided into type I and type II. ${ }^{61}$ Type I is characterized by spinal anterior horn involvement and death in infancy, and consequently will not be addressed in this review. ${ }^{61}$ Type II pontocerebellar atrophy is characterized by progressive microcephaly and severe cognitive and motor delays, in addition to dyskinesia and dystonia. ${ }^{61}$ It is noteworthy that some authors classify $\mathrm{PCH}$ as a degenerative disorder rather than a true malformation. ${ }^{62}$

In a chart review of 24 children with PCH type II, significant developmental and language delays were reported in all children. ${ }^{60} \mathrm{~A}$ series of case reports have also been published $^{63-65}$ in which all four children presented with developmental delay and two were reported to have language deficits. ${ }^{63}$ Neurological findings included microcephaly in all children, ${ }^{60,63-65}$ seizures $(25 \%),{ }^{60}$ respiratory abnormalities $(46 \%),{ }^{60}$ hypotonia $(16 \%),{ }^{60,65}$ hypertonia $(13 \%),{ }^{60,63,64}$ oculomotor anomalies, ${ }^{63}$ ataxia $(8 \%),{ }^{60}$ and dyskinetic and choreic movements (58\%). ${ }^{60,64,65}$

Associated CNS malformations including ventriculomegaly and reduced white matter were present in about half of the children described from chart review, ${ }^{60}$ ventricular and sulci widening and myelination delay in two children in a case report, ${ }^{64}$ and thinning of the corpus callosum in one. ${ }^{64}$ Facial dysmorphism and orthopaedic anomalies were described in one child. ${ }^{63}$
Overall, PCH type II is associated with significant global developmental delays and neurological deficits.

\section{Cerebellar agenesis}

Cerebellar agenesis is characterized by a complete or nearcomplete absence of the cerebellum ${ }^{66}$ (Fig. 6). Very few studies have described the outcome of children with cerebellar agenesis. Titomanlio et al. ${ }^{67}$ presented a case of a 17 -year-old male with isolated cerebellar agenesis. Mild cognitive impairment, ataxia, and dysmetria were documented. However, no standardized outcome measures were used. On the other hand, near-total absence of the cerebellum was reported in five children. ${ }^{68}$ All children had developmental delay, including one with severe developmental delay. Moreover, $100 \%$ of children had delayed language development. Only one case was reported to have associated cerebral malformations, however they were not further described. ${ }^{68}$

\section{DISCUSSION}

Cerebellar malformations are now diagnosed with increasing frequency in the fetal and neonatal period. ${ }^{69,70}$ As such, the importance of accurate prognostic information to guide parental decision making has become essential. However, despite recent advances in neuroimaging and the growing interest in the role of the cerebellum in higherorder cognitive functions, our review of the literature

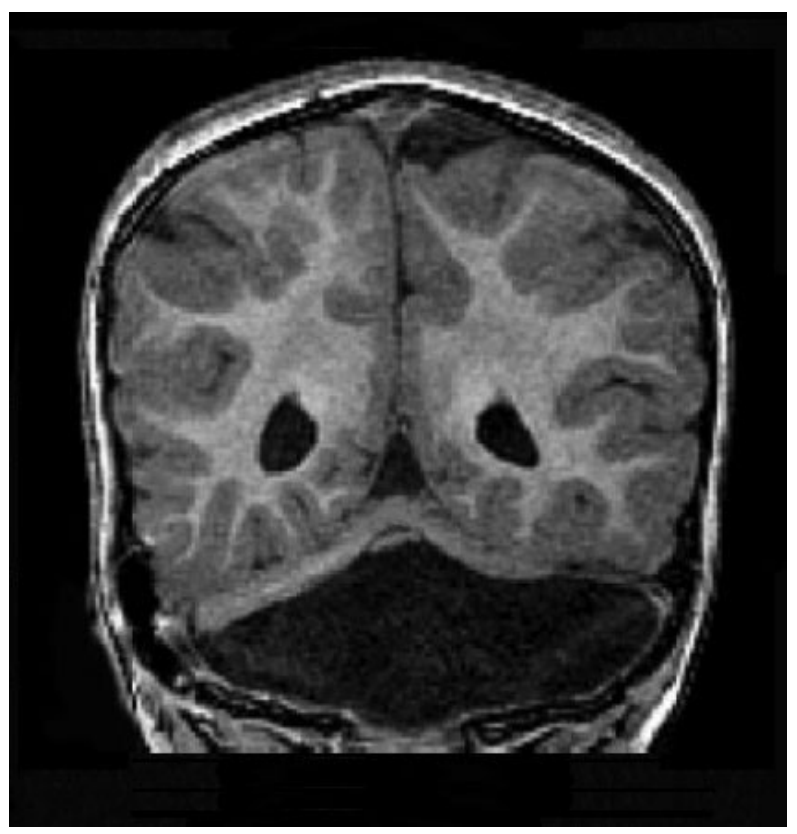

Figure 6: T1-weighted coronal magnetic resonance image representing near-complete absence of the cerebellum in an 18-month-old child. 
suggests that the neurodevelopmental and functional outcome of children with cerebellar malformations remains poorly defined. Important inconsistencies in the outcomes reported are frequent, and the spectrum of disability is often broad, ranging from normal or near-normal to profound disability for a given malformation. Furthermore, the results of this comprehensive review show that clear diagnostic criteria for the different types of cerebellar malformations are lacking, resulting in the description of heterogeneous study populations with results that are not easily generalizable.

However, certain global trends in the neurodevelopmental outcome in survivors of cerebellar malformations were evident. Overall, children with MCM and isolated IVH show good developmental progress, whereas children with molar tooth sign/Joubert syndrome, vermis hypoplasia, PCH type II, and cerebellar agenesis are likely to experience moderate to severe global developmental delay. Outcome data remain conflicting in children with DWM; however, the presence of a normally lobulated vermis and the absence of associated CNS anomalies appear to be associated with a better neurodevelopmental outcome. Finally, patients with isolated cerebellar hypoplasia (not including the vermis) appear to have a more favourable prognosis.

Our review over the 10-year period underscored wideranging outcomes in children with cerebellar malformations. These inconsistencies can be partly explained by the lack of a widely accepted classification scheme for cerebellar malformations. For example, several studies collapsed different diagnostic groups and described the developmental outcome of survivors collectively. Consequently, children with different types of cerebellar malformations were often clustered together, which further impeded our understanding of the relative contribution of individual cerebellar diagnostic groups on subsequent neurodevelopmental disabilities. Furthermore, the reported wide-ranging outcomes identified in the current review may also be attributed to the overall lack of rigorous study designs and standardized outcome measures, where a prominent $83 \%$ of the reviewed studies were conducted retrospectively. Consequently, children with normal development or mild impairments may be underrepresented. Moreover, just under half (39\%) of the reviewed studies were case reports or case series. The complete absence of any longitudinal data over this 10 -year review period is quite striking. Longitudinal studies are essential, particularly in assessing and monitoring children's progress during important developmental transitions through the lifespan. Furthermore, the wide age range at testing introduced a lot of noise in the studies, and consequently the appreciation of specific outcome information at key intervals in child development was limited.
It is noteworthy that the lack of standardized assessment tools in $74 \%$ of the studies reviewed was also an important limitation of the current literature. Furthermore, the studies focused primarily on mortality and morbidities such as IQ, neurological impairments, and other biomedical markers. Cognitive, language, social, and behavioural disabilities were seldom investigated. Given the growing evidence supporting an important role of the cerebellum in cognitive function, including language, perception, and social skills, outcome measures used to date prove to be largely insufficient in this population. ${ }^{10}$ Additionally, measures of quality of life and parental burden were completely absent in our 10 -year review. These are essential in capturing the added impact of these malformations in the child and their family.

\section{LIMITATIONS}

Several limitations of our review should be highlighted. First, it was limited to English-language literature, and therefore studies published in other languages were not included. Second, given that there is no universally accepted classification scheme for posterior fossa malformations, the diagnostic categories proposed by the different studies in this review often varied from one study to another. Moreover, cerebellar diagnostic groups were at times collapsed by some authors because of a small sample size. Consequently, it is likely that certain aspects of the outcome data described in this review may not reflect accurately the developmental outcome of survivors of cerebellar malformations. Finally, given that our review extended over a 10 -year study period, recent advances in genetics and neuroimaging studies that have permitted a more accurate identification of cerebellar malformations and their associated chromosomal anomalies are not necessarily reflected in the current paper.

\section{CONCLUSION}

Rigorous longitudinal outcome studies that incorporate advanced neuroimaging techniques and genetic testing are urgently needed to delineate better the long-term significance of cerebellar malformations. Furthermore, more holistic measures that assess a larger number of functional domains are required in order to capture the entire spectrum of disability in children with cerebellar malformations that extends far beyond the motor and cognitive domains. These measures must not be limited to evaluating impairments but extended to their functional impact on daily activities, school performance, and societal roles. Collectively, such studies will assist in the development of a rational and clinically useful classification and ultimately improve our understanding of the functional consequences of cerebellar malformations at key intervals across the lifespan. 
Finally, a better understanding of the developmental and functional consequences of cerebellar malformations on the developing child will allow earlier and possibly more effective therapeutic interventions, since cerebellar development is not fully completed before the end of the first postnatal years. ${ }^{49}$ Additionally, given the greater plasticity of the younger brain coupled with the highly plastic properties of the cerebellum, ${ }^{71}$ early targeted intervention could potentially translate into reorganization of the cerebellar circuitry and result in improved outcome.

\section{REFERENCES}

1. Boltshauser E. Cerebellum-small brain but large confusion: a review of selected cerebellar malformations and disruptions. Am 7 Med Genet 2004; 126A: $376-85$.

2. Sandalcioglu IE, Gasser T, van de Nes JAP, Menken U, Stolke D, Wiedemayer H. Fusion of the cerebellar hemispheres ventral to the brainstem: a rare hindbrain-related malformation. Childs Nerv Syst 2006; 22: 73-77.

3. Metropolitan Atlanta Congenital Defects Program, 2005.

4. Ecker JL, Shipp TD, Bromley B, Benacerraf B. The sonographic diagnosis of Dandy-Walker and Dandy-Walker variant: associated findings and outcomes. Prenat Diag 2000; 20: 328-32.

5. Forzano F, Mansour S, Ierrullo A, Homfray T, Thilaganathan B. Posterior fossa malformation in fetuses: a report of 56 further cases and a review of the literature. Prenat Diag 2007; 27: 495-501.

6. Steinlin M, Styger M, Boltshauser E. Cognitive impairments in patients with congenital nonprogressive cerebellar ataxia. Neurology 1999; 53: 96673.

7. Middleton FA, Strick PL. Cerebellar projections to the prefrontal cortex of the primate. 7 Neurosci $2001 ; 21$ : 700-12.

8. Tamada T, Miyauchi S, Imamizu H, Yoshioka T, Kawato M. Cerebrocerebellar functional connectivity revealed by the laterality index in tool-use learning. Neuroreport 1999; 10: 325-31.

9. Schmahmann JD, Pandya DN. The cerebrocerebellar system. Int Rev Neurobiol 1997; 41: 31-60.

10. Schmahmann JD, Sherman JC. The cerebellar cognitive affective syndrome. Brain Dev 1998; 121: 561-79.

11. Schmahmann JD. Disorders of the cerebellum: ataxia, dysmetria of thought, and the cerebellar cognitive affective syndrome. 7 Neuropsychiatry Clin Neurosci 2004; 16: 367-78.

12. Schmahmann JD, Weilburg JB, Sherman JC. The neuropsychiatry of the cerebellum - insights from the clinic. Cerebellum 2007; 6: 254-67.

13. Gordon N. The cerebellum and cognition. Eur 7 Paediatr Neurol 2007; 11: $232-34$.

14. Barkovich AJ. Pediatric neuroimaging. Fourth edn. Philadelphia: Lippincott Williams \& Wilkins, 2005.

15. Barkovich AJ, Kjos BO, Norman D, Edwards MS. Revised classification of posterior fossa cysts and cystlike malformations based on results of multiplanar MR imaging. Am 7 Neuroradiol 1989; 10: 977-88.

16. Parisi MA, Dobyns WB. Human malformations of the midbrain and hindbrain: review and proposed classification scheme. Mol Genet Metab 2003; 80: $36-53$.

17. Boddaert N, Klein O, Ferguson N, et al. Intellectual prognosis of the Dandy-Walker malformation in children: the importance of vermian lobulation. Neuroradiology 2003; 45: 320-24.

18. Kumar R, Jain MK, Chhabra DK. Dandy-Walker syndrome: different modalities of treatment and outcome in 42 cases. Childs Nerv Syst 2001; 17: 348-52.
19. Aletebi F, Fung K. Neurodevelopmental outcome after antenatal diagnosis of posterior fossa abnormalities. F Ultrasound Med 1999; 18: 683-89.

20. Klein O, Pierre-Kahn A, Boddaert N, Parisot D, Brunelle F. DandyWalker malformation: prenatal diagnosis and prognosis. Childs Nerv Syst 2003; 19: 484-89.

21. Kölble N, Wisser J, Kurmanavicius J, et al. Dandy-Walker malformation: prenatal diagnosis and outcome. Prenat Diag 2000; 20: 318-27.

22. Has R, Ermis H, Yüksel A, et al. Dandy-Walker malformation: a review of 78 cases diagnosed by prenatal sonography. Fetal Diagn Ther 2004; 19: $342-47$.

23. Poot M, Kroes H, v d Wijst SE, et al. Dandy-Walker complex in a boy with a $5 \mathrm{Mb}$ deletion of region $1 \mathrm{q} 44$ due to a paternal $\mathrm{t}(1 ; 20)(\mathrm{q} 44 ; \mathrm{q} 13.33)$. Am 7 Med Genet 2007; 143a: 1038-44.

24. Abdel-Salam GMH, Shehab M, Zaki MS. Isolated Dandy-Walker malformation associated with brain stem dysgenesis in male sibs. Brain Dev 2006; 28: 529-33.

25. Long A, Moran P, Robson S. Outcome of fetal cerebral posterior fossa anomalies. Prenat Diag 2006; 26: 707-10.

26. Limperopoulos C, Robertson RL, Estroff JA, et al. Diagnosis of inferior vermian hypoplasia by fetal magnetic resonance imaging: potential pitfalls and neurodevelopmental outcome. Am 7 Obstet Gynecol 2006; 194: 1070-76.

27. Haimovici JA, Doubilet PM, Benson CB, Frates MC. Clinical significance of isolated enlargement of the cisterna magna $(>10 \mathrm{~mm})$ on prenatal sonography. 7 Ultrasound Med 1997; 16: 731-34.

28. Zimmer EZ, Lowenstein L, Bronshtein M, Goldsher D, Aharon-Peretz J. Clinical significance of isolated mega cisterna magna. Arch Gynecol Obstet 2007; 276: 487-490.

29. Maria BL, Bozorgmanesh A, Kimmel KN, Theriaque D, Quisling RG. Quantitative assessment of brainstem development in Joubert syndrome and Dandy-Walker syndrome. 7 Child Neurol 2001; 16: 751-58.

30. Kumandas S, Akcakus M, Coskun A, Gumus H. Joubert syndrome: review and report of seven new cases. Eur F Neurol 2004; 11: 505-10.

31. Valente EM, Brancati F, Silhavy JL, et al. AHI1 gene mutations cause specific forms of Joubert syndrome-related disorders. Ann Neurol 2006; 59: 527-34.

32. Fennell EB, Gitten JC, Dede DE, Maria BL. Cognition, behavior, and development in Joubert syndrome. 7 Child Neurol 1999; 14: 592-96.

33. Maria BL, Quisling RG, Rosainz LC, et al. Molar tooth sign in Joubert syndrome: clinical, radiologic, and pathologic significance. 7 Child Neurol 1999; 14: 368-76.

34. Romano S, Boddaert N, Desguerre I, et al. Molar tooth sign and superior vermian dysplasia: a radiological, clinical and genetic study. Neuropediatrics 2006; 37: 42-45.

35. Gitten J, Dede D, Fennell E, Quisling R, Maria BL. Neurobehavioral development in Joubert syndrome. 7 Child Neurol 1998; 13: 391-97.

36. Kumar J, Kumar A, Saha S. The molar tooth sign of Joubert syndrome. Arch Neurol 2007; 64: 602-3.

37. Hodgkins PR, Harris CM, Shawkat FS, et al. Joubert syndrome: long-term follow-up. Dev Med Child Neurol 2004; 46: 694-99.

38. Torres M, Buceta M, Cajide M. Development of a child with Joubert syndrome. Span 7 Psychol 2001; 4: 72-78.

39. Ray J, Majumder AG, Das D, Mukhopadhyay D, Mondol S. Joubert syndrome: a major brain malformation. F Indian Med Assoc 2007; 105: 392-94.

40. Steinlin M, Schmid M, Landau K, Bolthauser E. Follow-up in children with Joubert syndrome. Neuropediatrics 1997; 28: 204-11.

41. Braddock BA, Farmer JE, Iverson JM, Maria BL. Oromotor and communication findings in Joubert syndrome: further evidence of multisystem apraxia. 7 Child Neurol 2006; 21: 160-73.

42. Chemli J, Abroug M, Tlili K, Harbi A. Rhombencephalosynapsis diagnosed in childhood: clinical and MRI findings. Eur 7 Pediatr Surg 2007; 11: 35-38. 
43. Toelle SP, Yalcinkaya C, Nocer N, et al. Rhombencephalosynapsis: clinical findings and neuroimaging in 9 children. Neuropediatrics 2002; 33: 209-14.

44. Utsunomiya H, Takano K, Ogasawara T, Hashimoto T, Fukushima T, Okazaki M. Rhombencephalosynapsis: cerebellar embryogenesis. Am 7 Neuroradiol 1998; 19: 547-49.

45. Aydingoz U, Cila A, Aktan G. Rhombencephalosynapsis associated with hand anomalies. Br f Radiol 1997; 70: 764-66.

46. Danon O, Elmaleh M, Boutakobza B, Fohlen M, Hadjnacer K, Hassan M. Rhombencephalosynapsis diagnosed in childhood: clinical and MRI findings. Magn Reson Imaging 2000; 18: 99-101.

47. Jellinger KA. Rhombencephalosynapsis. Acta Neuropathol 2002; 103: 305-6.

48. Odemis E, Cakir M, Aynaci FM. Rhombencephalosynapsis associated with cutaneous pretibial hemangioma in an infant. 7 Child Neurol 2003; 18: $225-28$.

49. ten Donkelaar H, Lammens M, Thijssen HOM, Renier WO. Development and developmental disorders of the human cerebellum. 7 Neurol 2003; 250: $1025-36$.

50. Patel S, Barkovich AJ. Analysis and classification of cerebellar malformations. Am 7 Neuroradiol 2002; 23: 1074-87.

51. Soto-Ares G, Delmaire C, Deries B, Vallee L, Pruvo JP. Cerebellar cortical dysplasia: MR findings in complex entity. Am 7 Neuroradiol 2000; 21: 1511-19.

52. Wassmer E, Davies P, Whitehouse WP, Green SH. Clinical spectrum associated with cerebellar hypoplasia. Pediatr Neurol 2003; 28: 347-51.

53. Yapici Z, Eraksoy $M$. Non-progressive congenital ataxia with cerebellar hypoplasia in three families. Acta Paediatr 2005; 94: 248-53.

54. Tavano A, Grasso R, Gagliardi C, et al. Disorders of cognitive and affective development in cerebellar malformations. Brain 2007; 130: 2646-60.

55. McCollom D, Rashidian J. Prenatal diagnosis of unilateral cerebellar hypoplasia. F Diagn Med Sonography. 2003; 19: 120-23.

56. Ventura P, Presicci A, Perniola T, Campa MG, Margari L. Mental retardation and epilepsy in patients with isolated cerebellar hypoplasia. 7 Child Neurol 2006; 21: 776-81.
57. Tavano A, Fabbro F, Borgatti R. Language and social communication in children with cerebellar dysgenesis. Folia Phoniatr Logop 2007; 59: 201-9.

58. Bruck I, Antoniuk SA, De Carvalho Neto A. Cerebellar vermis hypoplasia non-progressive congenital ataxia. Arq Neuropsiquiatr 2000; 58: 897-900.

59. Koutsouraki E, Markou E, Karlovasitou A, Costa V, Baloyannis S. Clinical case: vermis hypoplasia with features of Smith-Lemli-Optiz syndrome. Int $\mathcal{Z}$ Neurosci 2007; 117: 443-51.

60. Steinlin M, Klein A, Haas-Lude K, et al. Pontocerebellar hypoplasia type 2: variability in clinical and imaging findings. Eur 7 Neurol 2007; 11: 146-52.

61. Barth PG. Pontocerebellar hypoplasia - how many types? Eur 7 Paediatr Neurol 2000; 4: 161-62.

62. Barth PG, Aronica E, de Vries L, et al. Pontocerebellar hypoplasia type 2: a neuropathological update. Acta Neuropathol 2007; 114: 373-86.

63. Dilber E, Aynaci FM, Ahmetoglu A. Pontocerebellar hypoplasia in two siblings with dysmorphic features. 7 Child Neurol 2002; 17: 64-66.

64. Coppola G, Muras I, Pascotto A. Pontocerebellar hypoplasia type 2 (PCH2): report of two siblings. Brain Dev 2000; 22: 188-92.

65. Sans-Fito A, Campistol-Plana J, Mas-Salguero MJ, Poo-Arguelles P, Fernandez-Alvarez E. Pontocerebellar hypoplasia type 2 and Reye-like syndrome. 7 Child Neurol 2002; 17: 132-34.

66. Velioglu SK, Kuzeyli K, Ozmenoglu M. Cerebellar agenesis: a case report with clinical and MR imaging findings and a review of the literature. Eur $\mathcal{F}$ Neurol 1998; 5: 503-6.

67. Titomanlio L, Romano A, Del Giudice E. Cerebellar agenesis. Neurology 2005; 64: E21.

68. Gardner RJM, Coleman LT, Mitchell LA, et al. Near-total absence of the cerebellum. Neuropediatrics. 2001; 32: 62-68.

69. Limperopoulos C, du Plessis AJ. Disorders of cerebellar growth and development. Curr Opin Pediatr 2006; 18: 621-27.

70. Limperopoulos C, Robertson RLJ, Khwaja OS, et al. How accurately does current fetal imaging identify posterior fossa anomalies? Am $\mathcal{F}$ Roentgenol 2008; 190: 1637-43.

71. Chugani HT, Muller R-A, Chugani DC. Functional brain reorganization in children. Brain Dev 1996; 18: 347-56.

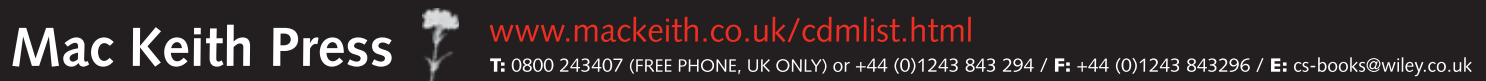

Developmental Disability and Ageing

A practical guide from Mac Keith Press

Gregory O'Brien and Lewis Rosenbloom

$240 \times 170 \mathrm{MM} / \mathrm{I} 44$ PAGES / SOFTBOUND / JANUARY 2009

$978-\mathrm{I}-898683-6 \mathrm{I}-2 / £ 20.00$
- Highly practical guide for clinicians and others involved in the care of ageing adults with developmental disabilities

- Boxes and summary tables present practical information concisely

- Individual cases illustrate the text 